\title{
Spectrum of Snake Bite in Bundelkhand Region
}

\author{
Rajat Jain¹, Rajiv Kumar², Swati Azad ${ }^{3}$, Gauri Naryani', Sanya Jain ${ }^{5}$ \\ ${ }^{1}$ Associate Professor, Department of Medicine, MLB Medical College, Jhansi, Uttar Pradesh, India. ${ }^{2}$ Assistant Professor \\ Department of TB and Chest, MLB Medical College, Jhansi, Uttar Pradesh, India. ${ }^{3}$ Medical Student, MLB Medical \\ College, Jhansi, Uttar Pradesh, India. ${ }^{4}$ Medical Student, MLB Medical College, Jhansi, Uttar Pradesh, India. ${ }^{5}$ Medical \\ Student, KIMS, Karad, Maharashtra, India.
}

\section{ABSTRACT}

\section{BACKGROUND}

Snake bite is a significant health concern, especially in rural populations of tropical and subtropical countries. Under-reporting of snake bite incidence and mortality is common. High mortality persists due to poor health facilities in rural areas and delayed reporting. WHO now recognizes snake bite as a neglected tropical disease. In India around 90\% snake bite are caused by the 'big four' - common Krait, Indian Cobra, Russell's Viper and Saw Scaled Viper. We wanted to study the spectrum of snake bite cases in tertiary care centre of Bundelkhand region.

\section{METHODS}

This is a retrospective descriptive study conducted in MLB Medical College, Jhansi. The data was collected from record files of patients who reported to the emergency department with the complaint of alleged snake bite between January 2018 to April 2019. Detailed history with reference to envenomation was obtained and time interval between bite and initiation of treatment was also noted. All data about clinical examination and blood investigations (including LFT, KFT, CBC, WBCT, ABG) were noted.

\section{RESULTS}

Male: female ratio was 1.53:1, with maximum number of patients in the age group of 31-40 years, followed by 21-30 years and 11-20 years. Majority of the cases reported in the season of monsoon. Most of the patients reported within 6 hours of getting snake bite (111 out of 225). 146 patients stayed till the completion of treatments while, 65 patients absconded and 12 left against medical advice for being kept under observation after symptoms were relieved. 2 patients expired due to respiratory failure.

\section{CONCLUSIONS}

High degree of suspicion of snakebite is required in patients who present with unexplained neurological manifestations, especially in monsoon season as in majority of patients there are no local manifestations at the site of bite. Special precautions are needed during monsoon and availability of plentiful anti-snake venom and facilities for artificial ventilation with fast ambulance service which can shift the patient to tertiary care centre is required. Most of the patients in Bundelkhand region reported within 6 hours to the tertiary care centre. Large number of patients at first consulted traditional healers or faith healers and it was only after the condition worsened that they reported to the hospital.

\section{KEY WORDS}

Snake Bite, WBCT, Neuro-Paralytic Manifestations

\author{
Corresponding Author: \\ Rajiv Kumar, \\ Assistant Professor, \\ Department of TB \& Chest, \\ MLB Medical College, \\ Jhansi, Uttar Pradesh, India. \\ E-mail: drrajivchest@gmail.com
}

DOI: $10.14260 /$ jemds/2019/607

Financial or Other Competing Interests: None.

How to Cite This Article:

Jain R, Kumar R, Azad S, et al. Spectrum of snake bite in Bundelkhand region. J. Evolution Med. Dent. Sci. 2019;8(36): 2798-2802, DOI:
Submission 25-06-2019,

Peer Review 20-08-2019,

Acceptance 28-08-2019,

Published 09-09-2019. 


\section{BACKGROUND}

WHO now recognizes snake bite as a neglected tropical disease. But India, which has the highest number of snake bite deaths, is apparently ill prepared for tackling the problem. According to India's national health profile (Published in 2018 ), there were 1,78,433 and 1,42,366 snake bite cases in 2016 and 2017 respectively with corresponding death figures of 1,063 and 948(1) as rural India struggles to get the adequate amount of antidotes and trained hands to tackle one of the commonest but neglected public health problem. Many victims never reach primary care facilities, and are therefore unreported. This is contributed to by socioeconomic and cultural factors that influence treatmentseeking behaviour with many victims opting for traditional practices rather than hospital care.(2) Inadequate treatment is a key factor behind such a high death toll. Most of the fatalities are due to the victim not reaching the hospital in time where definite treatment can be administered. In addition community is also not well informed about occupational risk and simple measures which can prevent the bite. It continues to adopt harmful first aid practices such as tourniquet, cutting and suction etc.

Though the various herbal remedies are scientifically unsubstantiated, yet they have been used for the longest time and still are continuously used. Reliance on traditional healers and myths further compound the problem. The primary health care centers lack sufficient ASV, emergency drugs and artificial ventilation facilities. Studies also reveal that primary care doctors do not treat snake bite patients mainly due to lack of confidence( ${ }^{3)}$ while, at the secondary and tertiary care level, multiple protocols are being followed for polyvalent anti-snake venom (ASV) administration, predominantly based on western textbooks. The time elapsed after the bite is of vital importance, because with the passage of time more venom gets bound to the tissues and is thus less manageable for neutralization by ASV. In India, common venomous snakes include the common krait (Bungarus caeruleus), the Indian cobra (Naja naja), Russelle's viper (Baboia Russelii), and Echis carinatus. (4)

Venomous snakes generally fall into 2 categories: snakes with fixed fangs usually use neurotoxic venom which affects the nervous system and breathing while other species have folded fangs which are only used when attacking prey or threats. Their toxin usually destroys skin tissue and causes internal bleeding.(4) Haemostatic abnormalities are the prima facie evidence of a viper bite. Cobras and kraits do not cause haemostatic disturbances.(1) Neurotoxicity is a key feature of some envenomations and acute neuromuscular weakness with respiratory involvement is the most clinically important neurotoxicity effect. We conducted a retrospective study on pattern of snake bite cases in tertiary care centre in Bundelkhand. The objective of this study is to investigate the morbidity and mortality of snake bite, observe clinical features, and analysis of the relationship between time delay, administration of ASV and progression of signs and symptoms.

\section{METHODS}

Our study was retrospective descriptive study conducted on 225 patients who presented to the emergency department of MLB Medical college, Jhansi with complain of alleged snake bite over a period of 16 months from January 2018 to April 2019. Ethical clearance was obtained from the institutional ethical committee for obtaining patient information from the record files. The data was collected from the record files of the patients who presented to the emergency department with a history of snake bite or showing evidence of envenomation such as swelling, bleeding manifestation, or neurotoxicity. Detailed history with reference to envenomation was obtained and time interval between bite and initiation of treatment was also noted. All data about clinical examination and blood investigations (Including LFT, $\mathrm{KFT}, \mathrm{CBC}, \mathrm{WBCT}, \mathrm{ABG}$ ) were noted.

\section{About 20 Minute WBCT \\ Twenty-minute whole blood clotting test $(20$ WBCT $)$ is considered as reliable test of coagulation which can be carried out by bedside(5) and is considered to be superior to 'capillary tube' method for establishing clotting capability in snake bite. A few milliliters of fresh venous blood should be placed in a fresh, clean and dry glass vessel preferably test tube and left undisturbed at ambient temperature for 20 minutes. After that tube should be gently tilted to detect whether blood is still liquid and if so then blood is incoagulable. The test should be carried out every 30 minutes from admission for 3 hours and then hourly after that.}

\section{RESULTS}

225 patients were included of which 136 were males and 89 were females so the male: female ratio is $1.53: 1$. Majority of the patient were in the age group of $31-40$ years (62 patients), followed by 21-30 years (60 patients), 11-20 years (52 patients), 41-50 years (26 patients), >50 years (20 patients) and then 0-10 years (5 patients). Maximum cases of snakebite were found to be in the monsoon season $(76$ cases out of 150), followed by summer ( 65 cases) and minimum in winter season (Only 9 cases). Most of the patients reported within 6 hours ( 72 cases) and between 6 to 24 hours ( 70 cases ) while, very few of the patients reported after more than 24 hours ( 5 cases ). Majority of the patients stayed in the hospital till to leave, while many of the patients either absconded before completion of treatment or left against medical advice. 2 of our patients expired due to respiratory failure (Because of delayed and severe presentation). Majority of patients stayed for up-to 2 days in the hospital. Those who required ventilation had to stay for more than 4 days. 106 patients presented with snake bite in lower limb, 101 in upper limb, 10 in head and neck while, 8 in neck. Majority of our patients had no local manifestations at the site of bite due to more prevalence of neurotoxic snakes. High degree of suspicious is required when unexplained 
neurological manifestations are seen in emergency department. Out of a total of 225 patients, $\mathrm{pH}$ value of 55 patients of paediatric age group could not be obtained. The neutrophil count was found to be normal in 45 patients and increased in 180 patients. 177 patients were found to be decreased in 177 patients and normal in 48 patients out of a total of 225 patients. 19 patients out of 225 had to be intubated because of deteriorating blood oxygen level.
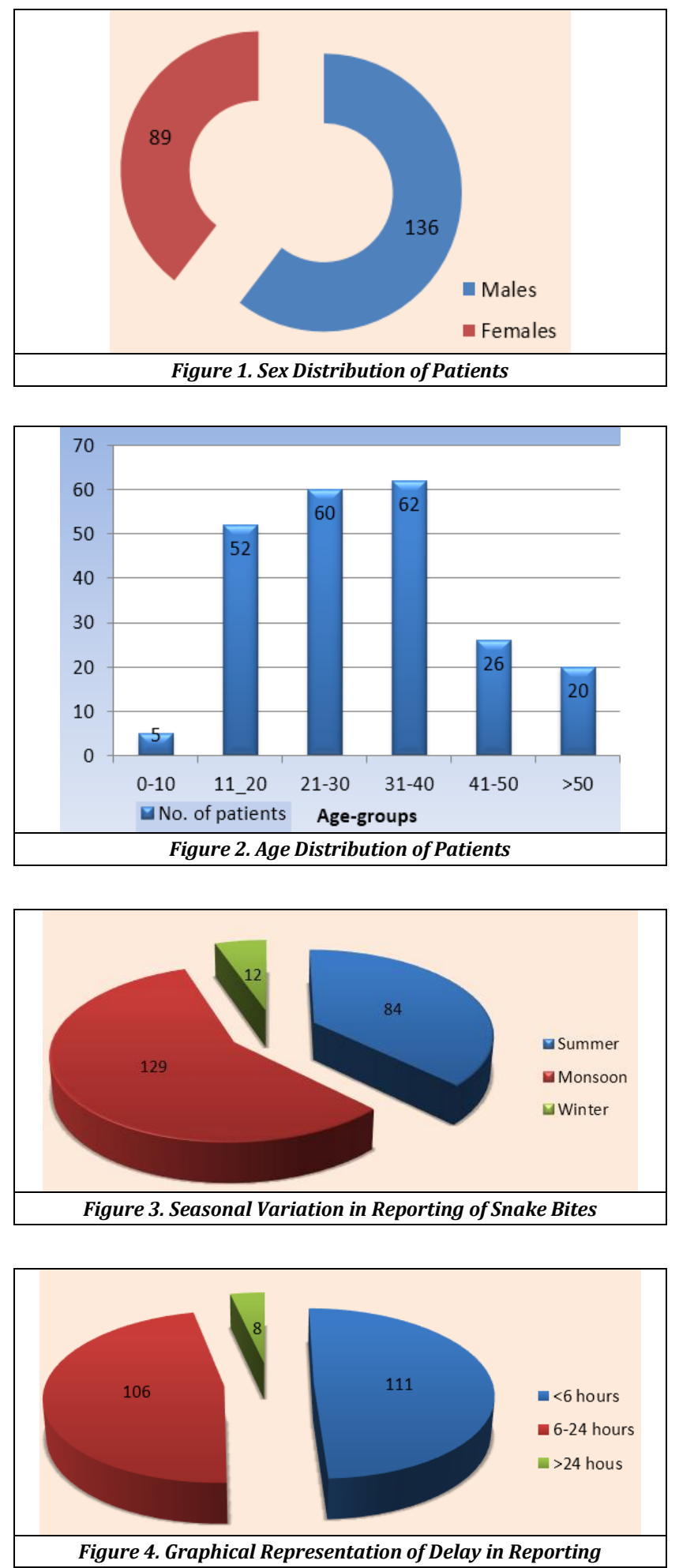
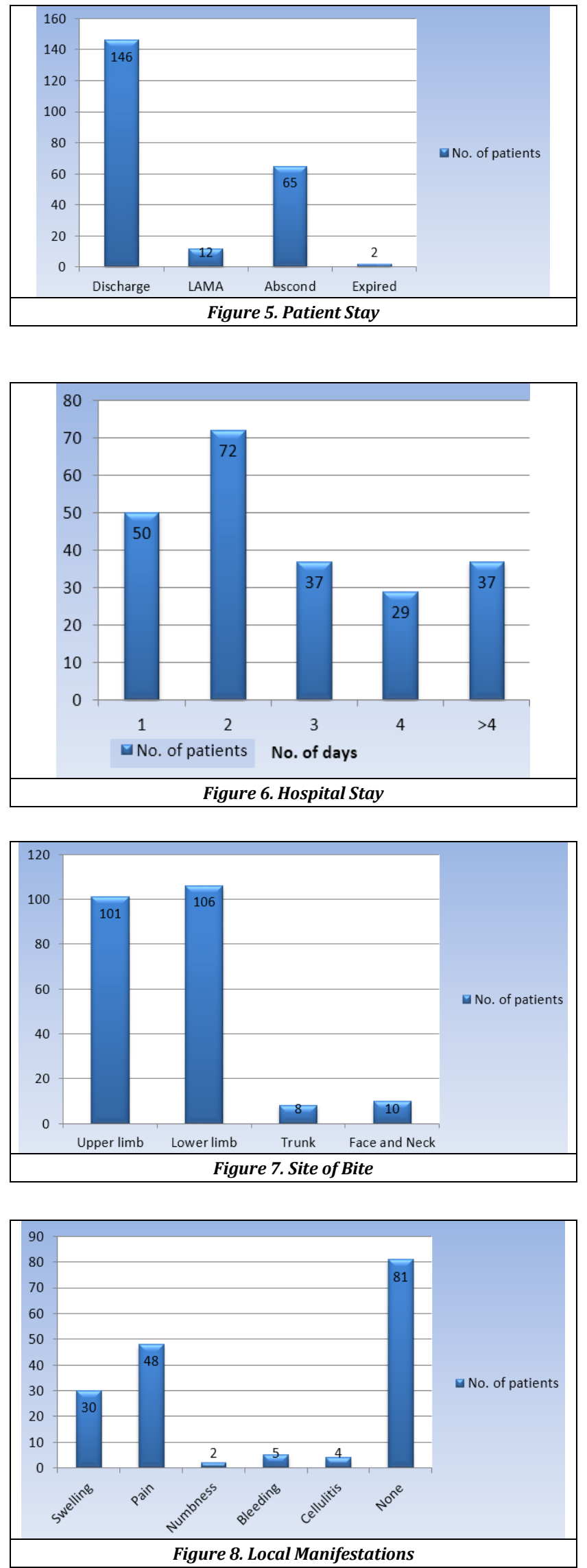

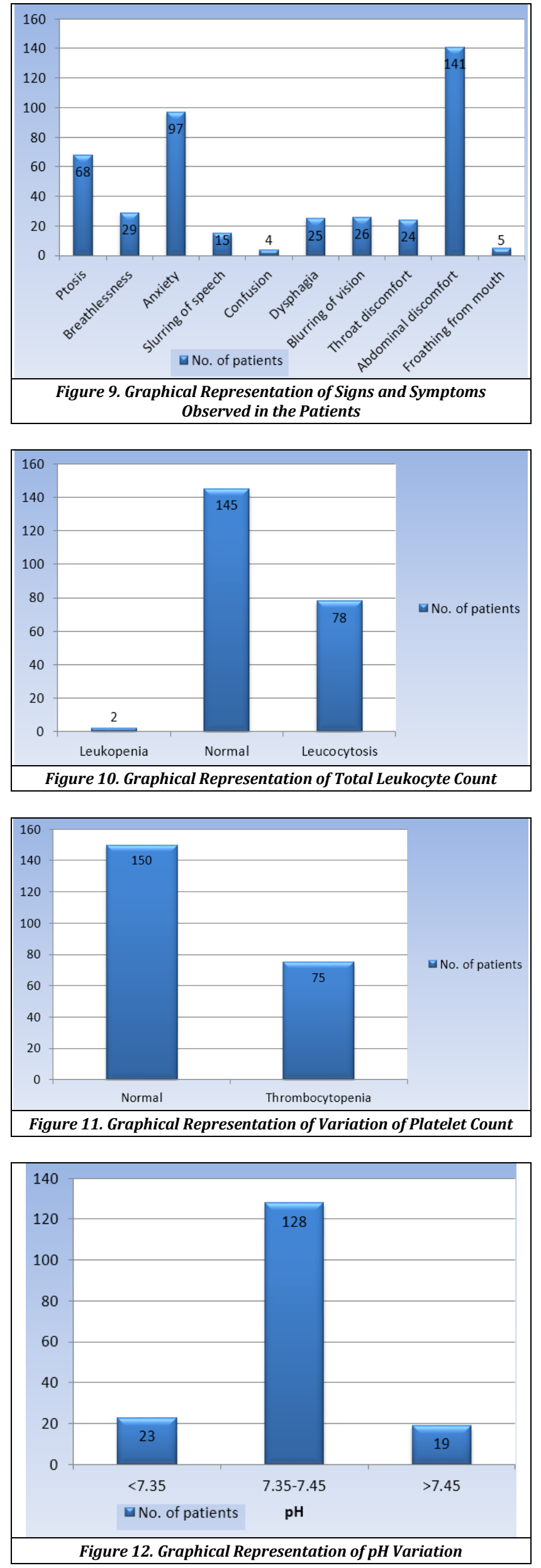

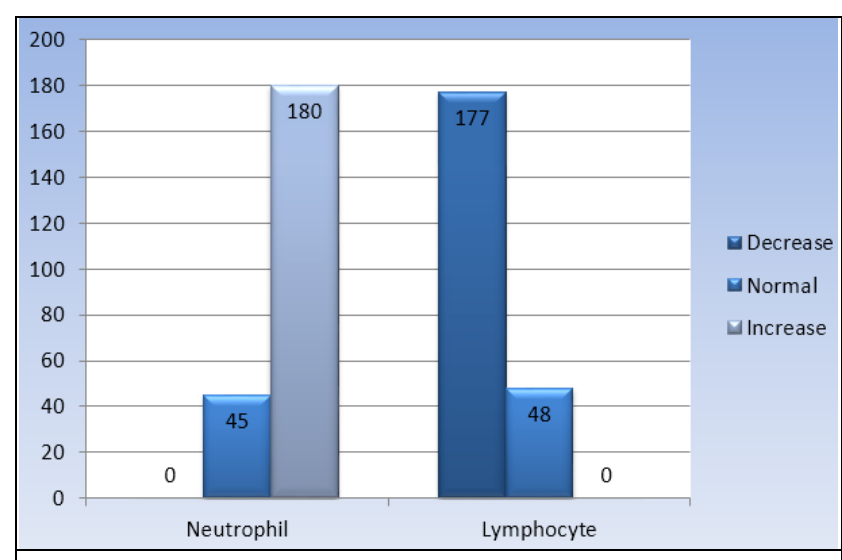

Figure 13. Graphical Representation of Differential Leukocyte Count
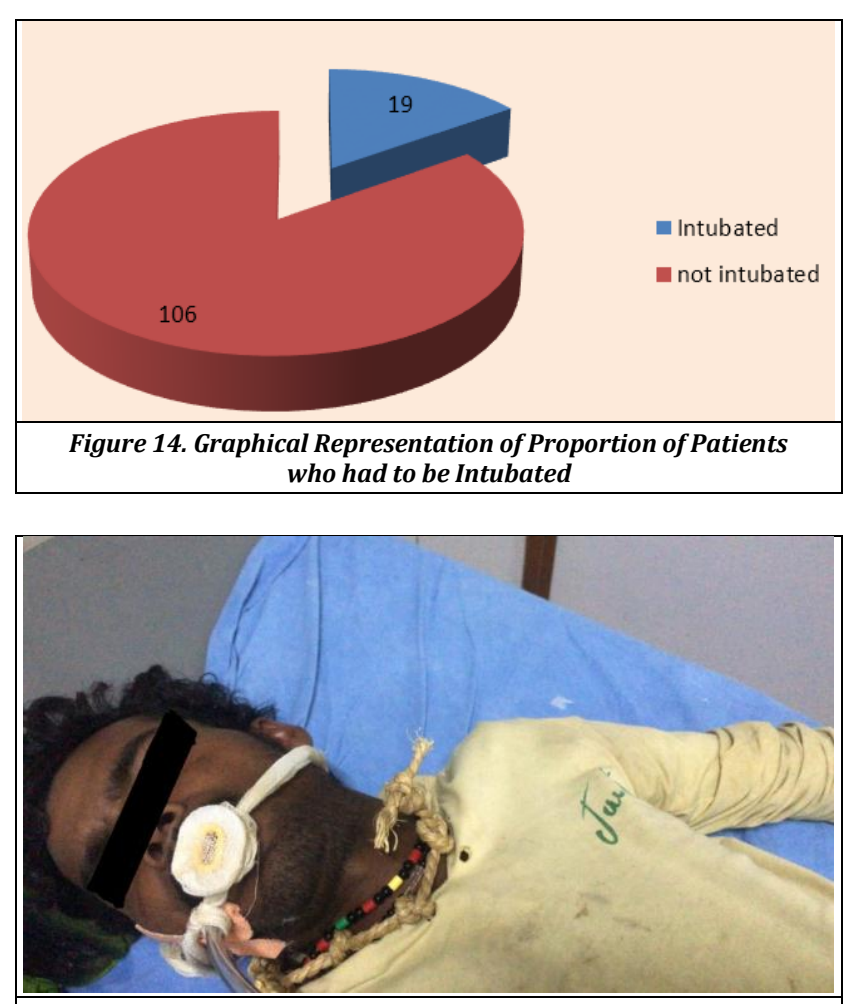

[A patient who Presented to Emergency Department of MLB Medical College, Jhansi. He had the Typical Rope Necklace, seen with most of Patients of Snake Bite in Bundelkhand Region who were at First taken to some Local People of Esoteric tradition (Tantriks)]

\section{DISCUSSION}

As expected, the number of male patients in the study was more than the number of female patients, as males in the rural areas are more into field work than females and the incidences of snake bite tend to be more in fields as compared to house.

Majority of patients were in the age group of $31-40$ years (62 patients), followed by 21 - 30 years ( 60 patients), $11-20$ years ( 52 patients), followed by 41 - 50 years ( 26 patients), then $>50$ (20 patients) and lastly those in the age group of 0 10 years ( 5 patients). Agricultural workers and children are the most affected. Children often suffer more severe effects than adults, due to their smaller body mass (2). The incidence of snake bite, as expected, were maximum in the season of monsoon (i.e. July, August, September) 129patients, followed by summer (i.e. March, April, May, June) 84 patients, then 
winter (October, November, December, January, February) 12 patients. Majority of the patients reported within 6 hours of snake bite (111 patients), followed by those within $6-24$ hours (106 patients), then after 24 hours ( 8 patients). The patients reporting between 6 - 24 hours either came from distant places or were referred from primary and secondary care centers.

Those reporting after 24 hours of snake bite (And also some of those reporting within 6 - 24 hour) included mostly those who at first visited some traditional healers or faith healers, had different kinds of ornaments made of rope and other materials and had applied some herbal medications over the site of bite. Among patients who reported, 146 stayed in the hospital until their treatment was completed. 72 patients left before the completion of treatment; 65 patients got absconded while 12 left against medical advice (LAMA) as soon as condition was stabilized.

2 patients expired due to respiratory failure. Both of these patients reported to the hospital with a delay of more than 6 hour (11 hours and 21 hours respectively) and could not be saved even after rigorous efforts including administration of ASV and intubation as per the requirement. Among the patients who stayed in the hospital until asked to leave (i.e. 146 patients), 50 patients, who did not suffer any complications, were discharged after being kept under observation for approx. 24 hours, 72 patients had to stay for 2 days, 37 of them had to stay for 3 days, 29 for 4 days and 37 for more than 4 days for complete treatment. 106 patients presented with snake bite in lower limb, 101 in upper limb, 8 in trunk and 12 in neck or face. The bites in lower limb are usually accidental as when snakes are stepped upon while at other sites, could result due to sleeping on floor and open style habitation. 107 out of 225 patients did not present with any kind of local manifestations while, 40 had local swelling, 64 had pain at the site of bite, 2 had numbness, 7 had bleeding and 5 had cellulitis. Some patients also presented with cut marks (an attempt by the attendants to provide first aid to the patient by removing venom before reaching the hospital) at the site of bite.

Ptosis was present in 88 patients, breathlessness in 38 , anxiety in 125 , slurring of speech in 19 , confusion in 5 , dysphagia in 32 , blurring of vision in 34 , throat discomfort (Including choking sensation) in 31, abdominal discomfort (including pain abdomen, nausea, vomiting) in 182 and 6 patients presented with frothing from mouth. The high incidence of abdominal discomfort among the patients might suggest more prevalence of kraits( 6 ) in the Bundelkhand region. The total leukocyte count was found to be normal in 145 patients, increased in 78 patients and decreased in 2 patients. The differential leukocyte count indicated an increased neutrophil count in 180 patients while normal in 45 patients, and lymphocyte count to be decreased in 177 patients and normal in 48 patients. Neutrophil/ Lymphocyte ratio (NLR) is said to be a useful parameter as it increases significantly in patients developing complications to snake bite $^{(7) .}$

150 patients out of 225 had a normal platelet count (i.e. $1.65-4.15 \times 10^{6} / \mathrm{dL}$ while, 75 patients had a decreased platelet count. $\mathrm{pH}$ value was found to be normal in 128 patients, decreased in 23 and increased in 23 patients. This observation does not include all the patients because of unavailability of $A B G$ reports in patients of paediatric age group. 19 patients had to be intubated because of deterioration in oxygen saturation of blood. Acute neuromuscular weakness with respiratory involvement is the most clinically important neurotoxic effect.(8) So, artificial ventilation appears to be a key feature in increasing survival of patients with severe neuroparalytic features. 20-minute WBCT test was found to be negative in all patients except 1 .

This indicates that most of the snakes in Bundelkhand region are either non-poisonous or cause neuro-paralytic manifestations (Including cobras and kraits) while, snake bites causing vasculo-toxic manifestations (Caused by vipers) are not significantly found here.

\section{CONCLUSIONS}

High degree of suspicion of snakebite is required in patients who present with unexplained neurological manifestations, especially in monsoon season as in majority of patients there are no local manifestations at the site of bite. Special precautions are needed to be taken during monsoon and plentiful anti-snake venom, facilities for artificial ventilation with fast ambulance service to shift the patient to tertiary care centre are required. Most of the patients in Bundelkhand region reported within 6 hours to the tertiary care centre to receive proper treatment. Large number of patients at first consulted traditional healers or faith healers and it was only after the condition worsened that they reported to the hospital. Death rate due to snake bite is low in this institute, mainly due to availability of anti-snake venom, ABG and artificial ventilation. $20 \mathrm{~min}$. WBCT was found to be negative in all cases except 1 . There is higher prevalence of neurotoxic snakes than the vasculotoxic ones in Bundelkhand.

\section{REFERENCES}

[1] Kalyan R. India neglects snakebites despite 50K deaths each year. DH News services, Delhi: JAN 23, 2019.

[2] WHO fact sheet for snakebite envenoming, APRIL 8, 2019: p. 3.

[3] Singh S, Singh G. Snake bite: Indian guidelines and protocol. Medicine update of API. 2013: p. 424-6.

[4] Bawaskar HS, Bawaskar PH, Bawaskar PH. Snake bite in India: a neglected disease of poverty. The Lancet 2017;390(10106):1947-8.

[5] Simpson ID. Snakebite Management in India, the first few hours: a guide for primary care physicians. J Indian Med Assoc 2007;105(6):324, 326, 328 passim.

[6] Mehta SR, Sashindran VK. Clinical features and management of snake bite. Medical Journal Armed Forces India 2002;58(3):247-9.

[7] Elbey B, Baykal B, Yazgan UC, et al. The prognostic value of Neutrophil/ Lymphocyte ratio in patients with snake bites for clinical outcomes and complications. Saudi Journal of Biological Sciences 2017;24(2):362-6.

[8] Ranwaka UK, Lalloo DG, De Silva HJ. Neurotoxicity in snakebite - the limits of our knowledge. PLoS Negl Trop Dis 2013;7(10):e2302. 